Notfall Rettungsmed 2013 $\cdot 16: 92-92$

DOI 10.1007/s10049-012-1630-5

Online publiziert: 11. Juli 2012

๑) Springer-Verlag Berlin Heidelberg 2012

\author{
H. Moecke ${ }^{1}$ M. Reng ${ }^{2}$ - C.K. Lackner ${ }^{3}$ \\ ${ }^{1}$ Asklepios Institut für Notfallmedizin, c/o Asklepios Klinik St.Georg Hamburg \\ ${ }^{2}$ Medizinische Klinik II - Gastroenterologie, Goldberg-Klinik Kelheim GmbH \\ ${ }^{3}$ Healthcare Division, Drees \& Sommer, München
}

\title{
Infektionsschutzgesetz ohne Rettungsdienst
}

\section{Ein außergesetzlicher Notstand?}

Bei den Gesundheitsministern der

Das im letzten Jahr novellierte Infektionsschutzgesetz setzte wesentliche, richtungweisende Impulse, um die Rechtsgrundlagen für die Hygiene im Gesundheitswesen zu verbessern. Unter anderem wurde der Geltungsbereich des Gesetzes von den Krankenhäusern auch auf die Einrichtungen für ambulantes Operieren, auf Vorsorge- und Rehabilitationseinrichtungen, Dialyseeinrichtungen und Tageskliniken ausgeweitet. Das war richtig! Es stellt sich in diesem Zusammenhang jedoch die Frage, warum der gesamte Rettungsdienst, zu Boden und zu Luft, als wesentliche akutmedizinische Dienstleistung nicht explizit in den Geltungsbereich des Gesetzes eingeschlossen wurde.

Im Krankentransport und im Rettungsdienst ist der Umgang mit infektiösen Patienten inzwischen Alltag. Denn auf welche Weise gelangen die Patienten mit MRSA(methicillinresistenter Staphylococcus aureus)/ESBL- („extended-spectrum betalactamase")/KPC- (Klebsiella pneumoniae Carbapenemase) oder Noro-Virus-Infektionen in die Krankenhäuser oder später wieder zurück nach Hause, in eine Rehabilitationseinrichtung oder ins Pflegeheim? Oder wer bringt den Patienten mit Meningitisverdacht ins Krankenhaus?

Warum hat das Bundesgesundheitsministerium offenbar eine Hemmung, den Rettungsdienst als medizinische Dienstleistung wahrzunehmen, ihn als gesetzliche Leistung festzuschreiben? Weil es ein Länderthema ist? Weil in der Regel die Innenbehörden zuständig sind? Weil das Thema im Bundesministerium vergessen wurde oder weil die Bedeutung für den Rettungsdienst dort noch nicht ausreichend bekannt ist oder wahrgenommen wird?
Länder scheint der Rettungsdiensts besser wahrgenommen zu werden. In der Sitzung der Gesundheitsministerkonferenz der Länder am 30.06.2011 wurde mehrheitlich beschlossen, das BMG (Bundesministerium für Gesundheit) zu bitten, den Rettungsdienst als eigenes Leistungssegment im SGB (Sozialgesetzbuch) V zu verankern. So sagte der hessische Sozialminister Stefan Grüttner (CDU):

Es ist überfällig, dass der Rettungsdienst eine eigene Rechtsgrundlage für seine Leistungen tungsdienst muss endlich als ein eigenständiger Teil der gesetzlichen Leistung eingestuft werden. ... Es ist zum einen nicht mehr zeitgemäß, dass bei der Notfallrettung ausschließlich der Transport im Vordergrund steht. Dies ignoriert sträflich die seit Jahren bestehende Entwicklung der vorklinischen Versorgungsleitung und insbesondere der Notfallmedizin.

Ändern aber können die Bundesländer alleine nichts. Das BMG lehnte den hessischen Vorschlag ab, die Leistungen von Notarzt- und Rettungsdienst bleiben somit aus Bundessicht weiter exklusiv in der Landesgesetzgebung verortet.

Auf der Homepage des BMG gibt Bundesgesundheitsminister Daniel Bahr bekannt:

Ich habe selbst mal Nächte mit dem Rettungsdienst verbracht und erlebt, wie gut Notärzte und Rettungsassistenten zusammenarbeiten. (http://www.bmg.bund.de/ ministerium/presse/interviews/wir-setzendie-richtigen-anreize.html)

Wir stehen gerne bereit, ein paar Nächte mit dem Minister und seinen Beamten zu verbringen, um ihnen nahezubringen, dass Rettungsdienst eine medizinisch ärztliche im Sozialgesetzbuch V erhält. ... Der Ret-
Dienstleistung ist und nicht ein Transportunternehmen für liegende Passagiere. Die Arbeit von Notarzt- und Rettungsdienst muss endlich eine adäquate rechtliche Grundlage im SGB V erhalten.

Aus Sicht des Notarzt- und Rettungsdienstes sind für eine nachhaltige Entwicklung dringend 2 Dinge erforderlich:

1.die adäquate gesetzgeberische Verortung im SGB V - wie von den Ländern immer wieder gefordert,

2.die Entwicklung des politischen Bewusstseins, dass es sich bei der präklinischen Notfallmedizin um einen akutmedizinischen Sektor der Daseinsvorsorge handelt und nicht um ein Fahrtkostensegment.

Dann wäre es selbstverständlich, dass der Rettungsdienst beim Infektionsschutzgesetz und vergleichbaren Gesetzen mit berücksichtigt werden würde. Wir regen daher eine entsprechende Initiative des Deutschen Bundestages an. Den aus Bundessicht außergesetzlichen Notstand der Rettungsmedizin zu beseitigen, wäre eine echte gesetzliche Leistung.

\section{Korrespondenzadresse}

Prof. Dr. H. Moecke

Asklepios Institut für Notfallmedizin, c/o Asklepios Klinik St.Georg Hamburg Lohmühlenstraße 5, 20099 Hamburg h.moecke@asklepios.com

\section{Compliance with Ethics Guidelines}

Conflict of interest. H. Moecke, M. Reng and C.K. Lackner declare that they have no conflict of interest.

This article does not contain any studies with human or animal subjects. 